3 Drug-induced duodenal perforation in the paediatric patient with 4 thalassemia major, an unreported side effect of iron- chelating agent- a 5 case report

7 Fatima Tuz Zahra Shakir, Rizwan Sultan, Riaz Siddiqui, Mohammad Zubair 8 Shah, Ayesha Javed, Natasha Maryam

9 Department of General Surgery, Islamabad Medical Complex, Islamabad, Pakistan.

10 Correspondence: Fatima Tuz Zahra Shakir. Email: fatimatuzahra04@gmail.com

12 Abstract

13 Duodenal ulcer disease is uncommon in paediatric age group. Its perforation is even 14 rarer. However, it should be kept in mind when examining children with acute abdomen especially if there are signs of shock or possibility of upper gastrointestinal bleed. We report a case of a 6 years old female child, a known case of thalassemia major and taking oral Deferasirox since two years of age. She had atypical presentation as there was no previous history of peptic ulcer disease and she only suffered epigastric pain and vomiting for a week but due to lack of proper diagnosis at a local clinic developed duodenal ulcer perforation, which was ultimately diagnosed at

21 a tertiary care hospital and managed with Graham Patch Closure.

22 Keywords: Paediatric age group, duodenal ulcer, duodenal perforation, epigastric 23 pain, Desferasirox.

\title{
25 Introduction
}

26 Duodenal ulcer disease is common in adults whereas in children, its incidence is 27 1.55cases/year ${ }^{1}$. Its risk factors in adults are increased NSAID use, Helicobacter 28 pylori infection, cigarette smoking, alcohol abuse, physical and emotional stress. 
Medicine, stress and Helicobacter pylori infection are usually the cause of ulcers in paediatric age group. Perforation because of ulcers occurs in the first part of the duodenum, in its anterior wall. The patient can present with tachycardia, rigid abdomen, diminished bowel sounds, and in extreme cases shock with low urine output. Conversely, signs of toxicity may be absent in extreme age and immunecompromised people, which at times creates a diagnostic dilemma for the treating physician and surgeon.

Deferasirox is an iron-chelating agent which reduces liver iron concentration and serum ferritin levels. It has been available in the market since $2006^{1}$ and it is used in the management of Transfusional Haemosiderosis and Nontransfusion-Dependent Thalassemia ${ }^{2}$. Common gastrointestinal side effects are abdominal pain, nausea, vomiting, and diarrhoea ${ }^{2}$. Although well tolerated in most patients, there have been post-marketing reports of this drug causing GI ulceration and perforation in elderly patients ${ }^{3}$. Although very rare, we aim to create awareness regarding these complications in the pediatric age group.

\section{Case Report}

A six year old female child, known case of thalassemia major, presented to the emergency department of Islamabad Medical Complex in April 2021 with the complaint of epigastric pain for one week. Initially, it was mild in intensity and intermittent for which parents gave syrup Panadol. On the third day, the patient started suffering from poor oral intake, vomiting containing food contents, and no haematemesis. The parents took their child to a local clinic and got her managed with intravenous(IV) analgesia and IV fluids after which symptoms settled and they went home. The next day, she developed the same symptoms including epigastric pain and vomiting and took the same management from a local clinic. On the seventh day, the pain became unbearable for the child so the local clinic physician referred her to a tertiary care hospital. 
On examination, the patient was extremely irritable, uncooperative, and not lying straight due to severe abdominal pain. Heart rate $130 / \mathrm{min}$, respiratory rate:30/min, blood pressure: 90/60mmHg and saturation 92\% at room air. After IV analgesia when the patient allowed abdominal examination, it was found to be slightly distended with generalized tenderness, rigidity, rebound tenderness, and absent bowel sounds over one minute.

Past medical history revealed second cousin marriage among parents with thalassemia minor trait. At four months of age, she was diagnosed with thalassemia major, and then managed by a paediatrician on hyper-transfusion therapy and started with Deferasirox at the age of two years. She had regular visits with her paediatrician and the dose was adjusted according to body weight and the efficacy of the drug was monitored with serial liver function tests and Serum. Ferritin levels. She was not on any other medication and did not suffer from any previous gastrointestinal tract-related illness. Her recent blood transfusion was done earlier this month.

Relevant blood investigations revealed a total leukocyte count of 34500/cmm (normal: 4000-10000/cmm) with 92\% neutrophils (normal: 55-70\%), haemoglobin 12g/dl (normal: 12-16g/dl) with 35\% haematocrit (normal: 40-50\%), total bilirubin 4.1mg/dL (normal: 0.2-1.1mg/dL), alanine aminotransferase (AST) $172 \mathrm{U} / \mathrm{L}$ (normal: 5-34 U/L), aspartate aminotransferase (ALT) 194 U/L (normal: 5-50 U/L), alkaline phosphatase (ALP) $356 \mathrm{U} / \mathrm{L}$ (normal: 1 to 12years <500), sodium 132mmol/L (normal: 135-145 $\mathrm{mmol} / \mathrm{L}$ ), potassium $5.5 \mathrm{mmol} / \mathrm{L}$ (normal: $3.5-5.5 \mathrm{mmol} / \mathrm{L}$ ). Plain x-ray abdomen showed free gas under right hemidiaphragm (Figure.1).

Diagnosis of hollow organ perforation was established and after proper resuscitation in surgical ICU with IV fluids and acquiring urine output according to body weight, the patient underwent emergency laparotomy.

Per-operatively, purulent fluid was encountered in the peritoneal cavity (Figure.2: an arrow head pointing towards perforation). After exploration, about $5 \mathrm{~mm} \times 5 \mathrm{~mm}$ perforation was discovered in the anterior wall of the first part of the duodenum. 
85 Graham patch closure was performed after abdominal lavage. The d rain was placed 86 adjacent to patch repair.

The postoperative period was uneventful. She was tested for Helicobacter pylori serology which tested negative. Her Drain was removed on the $6^{\text {th }}$ postoperative day and then despite being negative on serology for Helicobacter pylori, she was discharged on triple therapy as a protocol for duodenal ulcer treatment (omeprazole, clarithromycin, and metronidazole). On $30^{\text {th }}$ day of follow-up, the patient was alive and tolerating oral diet well after Graham patch repair with no further complaints of epigastric pain.

\section{Discussion}

Peptic ulcer disease is extremely uncommon in children, and is not considered in differential diagnosis whenever dealing with upper abdomen pain. Due to lack of definitive diagnosis, the child is usually mismanaged which leads to perforation or bleeding of the ulcer. Anatomically, ulcer formation depends on intrinsic and extrinsic causes and can be primary and secondary. Primary ulcers are usually gastric in origin. Helicobacter pylori infection causes primary duodenal ulcers which are associated with infection of the gastric antral mucosa. Secondary ulcers are drug-induced; causes include increased NSAID and steroid intake. Stress-induced ulcers are also secondary due to traumatic delivery, burns, respiratory or cardiac distress, hypoglycaemia, sepsis, or dehydration in infants ${ }^{1}$. Fatal illness and trauma, ulcers associated with intracranial pathologies such as Cushing's ulcer or Curling's ulcer due to burns are causes in older children ${ }^{1}$. Secondary ulcers in children less than 10 years of age are usually silent and found in the duodenum and their initial manifestation as perforation, or bleeding is catastrophic. Gas under the diaphragm on x-ray is the fastest way to identify gut perforation , and exploratory laparotomy is the only way to reach a final diagnosis.

Deferasirox is an iron-chelating agent, vital in the management of Thalassemia Major. Abdominal pain, vomiting, nausea, and diarrhoea are common GI side effects ${ }^{2}$. It has a good safety profile when administered well within the required dose but there are post- 
114 marketing reports for upper GI perforation and haemorrhage due to ulceration ${ }^{3}$. Case

115 reports can be found after literature review reporting upper GI ulceration ${ }^{4}$ and 116 perforation ${ }^{5}$ in the elderly but so far only one case report has been documented besides 117 this one for perforation as a complication in the paediatric age group. Yadav. et al 118 reported a case of duodenal perforation in a child taking Deferasirox in $2013^{1}$ and 119 Bauters. et al reported a case of gastric ulcer in a 10 years old child on Deferasirox ${ }^{6}$ in 120 2010. The morbidity and mortality associated with this complication mandate for it to 121 be reported. World Health Organization has (WHO) developed the WHO-UMC 122 system for standardized case causality assessment for pharmaco-vigilance and adverse 123 drug reactions. Since our patient was not on any other medication and there is no 124 known association between peptic ulcer disease and beta-thalassemia major, our case 125 report is assessed as probable on WHO-UMC causality assessment scale ${ }^{7}$. Our suggestion is the use of proton pump inhibitors or antacids along with Deferasirox intake to prevent this life threatening drug induced side effect.

\section{Conclusion}

Duodenal perforation even though extremely rare in the pediatric age group should be suspected when dealing with acute abdomen to prevent morbidity and mortality. Careful surveillance is needed in patients taking iron -chelating agents such as Deferasirox and further research is required to study its probable relation in causing peptic ulcer disease.

Disclaimer: Abstract has not been previously presented or published in a conference.

137 The manuscript was not part of any research, Ph.D, or thesis project.

Conflict of Interest: Dr. Riaz Siddiqui is also a co-author who signed IRB Statement. Funding Sources: None to declare.

140 Patient's consent: Informed consent was taken from the patient's parents about this 141 case report. 


\section{References}

1. Yadav SK, Gupta V, El Kohly A, Al Fadhli W. Perforated duodenal ulcer: a rare complication of deferasirox in children. Indian $\mathrm{J}$ Pharmacol. 2013;45(3):293-4

2. Medscape.com. [cited 2021 Aug 18]. Available from: https://reference.medscape.com/drug/exjade-jadenu-deferasirox-343724.

3. Sciencedirect.com. 2021. Deferasirox - an overview | ScienceDirect Topics. [online] Available at: <https://www.sciencedirect.com/topics/medicine-anddentistry/deferasirox > [Accessed 8 April 2021].

4. Jencks, David MD; Kumar, Anita MD; Borum, Marie MD, EdD, MPH. A Rare Case of Deferasirox-induced Peptic Ulcer Disease. American Journal of Gastroenterology: 2017;112: S1415

5. Atay K, Eskazan T, Salihoglu A, Hatemi I. Fatal gastrointestinal bleeding and perforated duodenal ulcer: a rare complication of deferasirox in a patient with liver cirrhosis. Biomed Res (Aligarh) [Internet]. 2017 [cited 2021 Aug 19];28(12). Available from: https:/www.alliedacademies.org/articles/fatalgastrointestinal-bleeding-and-perforated-duodenal-ulcer-a-rare-complication-ofdeferasirox-in-a-patient-with-liver-cirrhosis.html

6. Bauters T, Mondelaers V, Robays H, Hunninck K, de Moerloose B. Gastric ulcer in a child treated with deferasirox. Pharm World Sci. 2010 Apr;32(2):1123.

7. The use of the WHO-UMC system for standardised case causality assessment [Internet]. World Health Organization. [cited 2021 Aug 21]. Available from: https://www.who.int/medicines/areas/quality_safety/safety_efficacy/WHOcausa lity_assessment.pdf. 


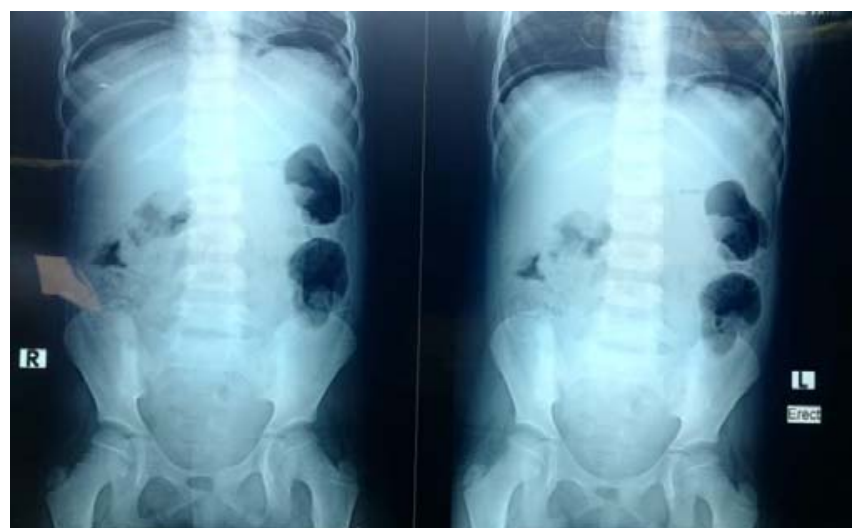

174

Figure 1: Plain x-ray abdomen showing free gas under right hemi diaphragm.

175

176
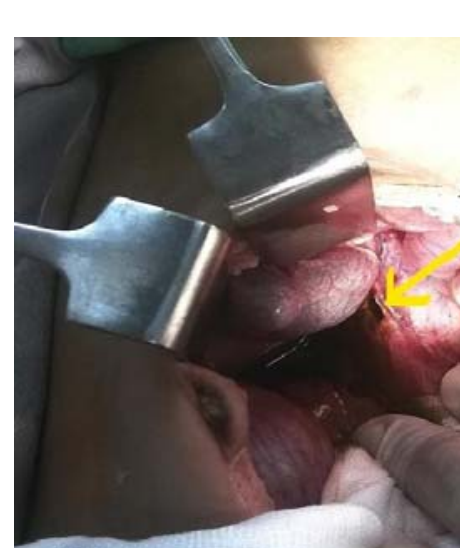

7..

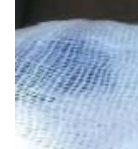
Figure 2: An arrow head pointing towards perforation 